\title{
Peritoneal Fluid and Serum Levels of Hepatocyte Growth Factor May Predict the Activity of Endometriosis
}

\author{
Khaleque Newaz Khan, ${ }^{\mathrm{a}}$ Hideaki Masuzaki, ${ }^{\mathrm{a}}$ Akira Fujishita, ${ }^{\mathrm{a}}$ Michio Kitajima, ${ }^{\mathrm{a}}$ Koichi Hiraki, ${ }^{\mathrm{a}}$ \\ Seiyou Miura, ${ }^{\mathrm{a}}$ Ichiro Sekine, ${ }^{\mathrm{b}}$ Tadayuki Ishimaru, ${ }^{\mathrm{a}}$ \\ aDepartment of Obstetrics and Gynecology, Nagasaki University School of Medicine, \\ bepartment of Molecular Pathology, Atomic Bomb Disease Institute, \\ Nagasaki 852-8501, Japan.
}

Running Headline: HGF in PF and serum of endometriosis

Address for Correspondence and Reprint Request:

Khaleque Newaz Khan, M.D, Ph.D

Department of Obstetrics and Gynecology

Nagasaki University School of Medicine

1-7-1 Sakamoto, Nagasaki 852-8501, Japan.

Tel: +81-95-849 -7363

Fax: +81-95-849-7365

E-mail: nemokhan@net.nagasaki-u.ac.jp 


\title{
Peritoneal Fluid and Serum Levels of Hepatocyte Growth Factor May Predict the Activity of Endometriosis
}

\begin{abstract}
Background: The scoring system and the activity of endometriosis in the peritoneal fluid (PF) and in intact tissue has been demonstrated. However, the suitable parameter in PF as well as in serum that may predict the activity of endometriosis is not well described. Therefore, we tried to examine the PF and serum concentrations of hepatocyte growth factor (HGF) in different revised American Society of Reproductive Medicine (r-ASRM) staging and morphologic appearances of endometriosis in an attempt to determine whether HGF can be clinically useful to predict the activity of pelvic endometriosis.

Methods: Peritoneal fluid was collected from 137 women with endometriosis and 57 women without endometriosis during laparoscopy and blood sampling was collected from 37 women with endometriosis and 21 women without endometriosis before laparoscopy. PF macrophages $(\mathrm{M} \phi)$ and endometrial stromal cells were isolated from six women with endometriosis and six control women in primary culture. The concentration of HGF in PF, serum and culture media was measured by enzyme-linked immunosorbent assay (ELISA). The changes in the levels of HGF in PF and serum according to different r-ASRM staging, morphologic appearances and phases of menstrual cycle were examined. The ability of isolated $\mathrm{M} \phi$ and stroma to secrete HGF in the culture media after stimulation with or without lipopolysaccharide (LPS) was evaluated.
\end{abstract}

Results: There was no difference in the PF and serum levels of HGF between women with stage I-II endometriosis and stage III-IV endometriosis. When we distributed serum levels of HGF according to different color appearances of endometriosis, we found a significantly higher serum levels of 
HGF in women containing dominant red peritoneal lesions in pelvic cavity $(740 \pm 109.3 \mathrm{pg} / \mathrm{mL})$ than those having other pigmented lesions (649 $\pm 79.5 \mathrm{pg} / \mathrm{mL}, \mathrm{p}<0.05$ ) or chocolate cysts $(485 \pm 43.1 \mathrm{pg} / \mathrm{mL}$, $\mathrm{p}<0.05$ ). Again, a significantly increased concentration of HGF in PF was found in women with endometriosis $(1451.75 \pm 90.7 \mathrm{pg} / \mathrm{mL})$ than that in non-endometriosis $(1120.5 \pm 77.3 \mathrm{pg} / \mathrm{mL}, \mathrm{p}<0.01)$ and in women harboring dominant red peritoneal lesions $(1685 \pm 183.4 \mathrm{pg} / \mathrm{mL})$ than that with other peritoneal lesions $(1224 \pm 67.8 \mathrm{pg} / \mathrm{mL}, \mathrm{p}<0.05)$ or chocolate cysts $(1118 \pm 83.1 \mathrm{pg} / \mathrm{mL}, \mathrm{p}<0.01)$. A higher HGF concentration in PF was observed in the menstrual phase of the cycle. Exogenous stimulation with LPS significantly increased the production of HGF in the culture media by M $\phi$ and stroma derived from women with endometriosis than that without endometriosis.

Conclusions: These results suggest that women with early or advanced endometriosis as measured by r-ASRM scoring system are not associated with an increase in either serum or PF concentrations of HGF. Rather HGF levels in serum and PF were significantly increased in women harboring blood-filled red peritoneal lesions and may be clinically useful to predict the activity of pelvic endometriosis.

Key words: Endometriosis, hepatocyte growth factor, peritoneal fluid, serum, lipopolysaccharide, disease activity 
Khan KN et al. Page 4

\begin{abstract}
Abbreviations
r-ASRM: revised-American Society of Reproductive Medicine; HGF: hepatocyte growth factor; PF: peritoneal fluid; ELISA: enzyme-linked immunosorbent assay; $\mathrm{M} \phi$ : macrophage; LPS: lipopolysaccharide.
\end{abstract}




\section{Introduction}

Hepatocyte growth factor (HGF) was first discovered as a mitogen for adult hepatocytes $(1,2)$

and is identical to scatter factor (3). Several lines of evidence have implied that HGF has mitogenic, motogenic and morphogenic functions in vitro on various epithelial cells derived from rodents and humans $(4,5)$. Recently, the presence of c-Met, the receptor of HGF on human endometrial epithelial cells and endothelial cells, has been reported (6). Synthesis of HGF by mesenchymal cells, coupled with demonstrated effects on epithelial and endothelial cells, suggests a paracrine mode of action (7). Furthermore, HGF has been shown to stimulate the proliferation, migration and morphogenesis of endometrial epithelial cell (8).

Besides sex steroid hormones, the growth of endometriosis can be regulated by different cytokines and growth factors in pelvic microenvironment (9-12). A line of evidence has already demonstrated that vascular endothelial growth factor (VEGF), HGF, and other cytokines are elevated in the peritoneal fluid (PF) of women with endometriosis and are associated with the growth and progression of endometriosis (13-16). However, most of these studies described a relationship between the changes in the concentrations of HGF, VEGF or other cytokines in the PF and advanced staging of endometriosis. The detail information regarding the changes in the PF levels of HGF in the different revised-ASRM staging and color appearances of women with pelvic endometriosis has been lacking. The only report by Zong LL et al. (17) described the parallel measurement of HGF concentration in serum and PF and distributed HGF results according to the r-ASRM staging of endometriosis. However, this study did not inform the relationship of HGF concentration in PF or serum with the color 
appearances of endometriosis.

An elevation in the PF concentrations of HGF, VEGF and interleukin-6 (IL-6) in advanced endometriosis have been reported recently $(15,16)$. Osuga et al. $(15)$ demonstrated that PF concentration of HGF in women with advanced endometriosis was significantly higher than those from women without endometriosis and found no difference from women with early endometriosis. However, both of these studies did not clarify the coexistence of colored lesions in pelvic cavity or chocolate cysts in their grouping of samples that could overestimate their results of VEGF or HGF in advanced endometriosis. The only report from our laboratory demonstrated that changes in the PF levels of HGF have an association with estradiol and both of them are elevated in the early stage of endometriosis and especially in those women with endometriosis harboring dominant distribution of highly active red peritoneal lesions (18).

Therefore, we tried to measure HGF concentrations in the PF of women with or without endometriosis and investigated their relationship in different revised-ASRM staging and morphologic appearances of endometriosis. With the speculation in mind that changes in the serum levels of HGF could be useful to predict the staging or activity of endometriosis, we also measured HGF in the serum of a fraction of these women with or without endometriosis. Finally, in an attempt to investigate the source of local production of HGF in pelvic environment, we examined the production of HGF by the basal and lipopolysaccharide (LPS) stimulated-macrophages (M $\phi)$ derived from the PF and endometrial stroma of women with or without endometriosis. 


\section{Materials and Methods}

Subjects. For the measurement of HGF in peritoneal fluid (PF group), a total of 137 women between 15 and 43 years of age undergoing laparoscopy either for pelvic pain, dysmenorrhea and/or for infertility were recruited in this study. A total of 57 women between 17-39 years of age who were free of endometriosis but operated for dermoid cyst or other benign ovarian tumors by laparoscopy formed the control group. We excluded women with uterine myoma or pelvic inflammatory disease as a control group who were investigated for infertility by laparoscopy and have a normal pelvis. Because, PF of these women contains a higher concentration of different macromolecules as found by our ongoing study or as described elsewhere $(19,20)$.

We tried to collect blood sampling from all these women before laparoscopy. However, a majority of these women refused to do so for their previous painful experience and/or for experimental purpose. Therefore, a parallel collection of sera was performed and stored from a part of these women who gave informed consent. We measured HGF in serum derived from these women, 37 women with endometriosis and 21 women without endometriosis (serum group). Since HGF can also be produced by blood cells, liver cells and kidney cells, we simultaneously performed hematology tests, liver function tests and renal function tests in all these women.

Endometriosis was diagnosed laparoscopically and histologically for all women in the study group. The extent of the disease was staged according to the revised-classification of American Society of Reproductive Medicine (revised-ASRM) (21). The distribution of patients in different revised-ASRM staging of endometriosis was as follows: serum group, stage I-II, n=19 and stage III-IV, n=18; PF group, 
stage I-II, n=75 and stage III-IV, n=62. Neither the study group nor the endometriosis free group had been on hormonal medication in the 3 months prior to the surgical procedure. All control women and women with endometriosis had regular menstrual cycles (28-32 days). The phase of the menstrual cycle was determined by histological dating of eutopic endometrium samples taken simultaneously with the peritoneal fluid samples. Menstrual dating was carried out by an independent pathologist. All induced menstrual cycles were excluded from the current study.

Peritoneal lesions of endometriosis were diagnosed by their macroscopic appearance according to published criteria (22) and categorized as red, black and white lesions as proposed in the latest revision of the ASRM classification (21). Depending on the translucency or transparency of the red lesions, we divided red peritoneal lesions into blood-filled opaque or nontransparent red lesions and non-opaque or transparent/translucent red lesions as already described recently (23). In this recent study, we demonstrated that opaque red lesions displayed the highest activity in women with endometriosis even when we isolated the presence of any single coexisting opaque red lesion from the women containing dominant black or white lesions. Therefore, the grouping of patients according to color appearance of endometriosis for our current study was done as follows: (1) women with red lesion, women containing a single opaque red lesion or dominant distribution of non-opaque red lesions; (2) women with combined black and white lesions, women containing dominant distribution of black or white lesions without existence of any opaque red lesion; (3) women with chocolate cysts, women containing variable sizes of chocolate cysts with scanty distribution of either black lesion or white lesion or both but without any evidence of red lesion. Peritoneal lesion or chocolate cyst in women with endometriosis was assessed by measuring the size of each lesion. This was confirmed by a second, blind observer from the photograph 
and video evidence of recorded file during laparoscopy as we reported previously $(18,23)$.

The distribution of women having different peritoneal lesions was as follows: serum group, red lesion, 11; combined black and white, 11; chocolate cysts, 15; PF group, red lesion, 36; combined black and white, 49; chocolate cysts, 44. This study was approved by the Institutional Review Board (IRB) of our University and informed consent was obtained from all women.

Collection of peritoneal fluid and serum. All visible PF was aspirated from the anterior and posterior cul-de-sac via a second puncture using a $4 \mathrm{~mm}$ metal cannula connected to an extension tube, before any internal manipulation and with the patient still in the horizontal supine position. The PF was transferred into sterile plastic tubes and kept on ice until arrival at the laboratory. The samples were then clarified by centrifugation at $1500 \mathrm{~g}$ for 10 minutes; the supematants were isolated and stored at $-70^{\circ} \mathrm{C}$ until assayed. Samples contaminated with blood from puncture sites were not included. Blood samples were collected from the women in the serum group before laparoscopy, centrifuged to collect sera and stored at $-70^{\circ} \mathrm{C}$ until assayed

HGF assay in peritoneal fluid and serum. All PF samples and sera were prospectively collected and assays were performed retrospectively. Peritoneal fluid and serum concentrations of HGF were measured in duplicate, using a commercially available sandwich enzyme linked immunosorbent assay (ELISA) developed by R \& D system in a blind fashion (Quantikine, R \& D system, Minneapolis, MN). The antibody used in HGF determination was raised against recombinant human HGF and do not cross-react with other cytokines. The limit of detection was $40.0 \mathrm{pg} / \mathrm{mL}$ for HGF. Both the intra-assay and inter-assay coefficients of variation were $<10 \%$ for this assay.

Collection of PF macrophages, endometrial and endometriotic stroma in primary 
culture. We collected stroma from the eutopic endometrium of six women with endometriosis and six women without endometriosis. Stroma was also isolated from six endometriotic lesions as collected from three women with pelvic endometriosis $(n=3)$ and from three chocolate cysts $(n=3)$. The procedures of isolation of $\mathrm{M} \phi$ and stroma in primary culture were described previously in detail (24-26). Briefly, Peritoneal fluid samples were centrifuged at $400 \mathrm{x}$ g for 10 minutes and the cellular pellet was under-layered with Lymphocyte Separation Medium (ICN, Aurora, OH) and centrifuged at $400 \mathrm{x}$ g for 10 minutes. Macrophages were collected from the interface and cultured in RPMI-1640 medium (GIBCO, Grand Island, NY) supplemented with $100 \mathrm{IU} / \mathrm{mL}$ of penicillin $\mathrm{G}, 50 \mathrm{mg} / \mathrm{mL}$ of streptromycin, $2.5 \mu \mathrm{g} / \mathrm{mL}$ of amphotericin $\mathrm{B}$ and $10 \%$ fetal bovine serum at $37^{\circ} \mathrm{C}$ in $5 \% \mathrm{CO}_{2}$ in air.

The macrophages were allowed to adhere to the culture plate for 2 hours, after which the non-adherent cells were removed by washing the plates three times with RPMI medium. The adherent cells remaining on the plates were more than 95\% macrophages as determined by their morphology and by immunocytochemical staining using CD68 (KP1), a mouse monoclonal antibody from Dako, Denmark. The cells used for immunocytochemical staining were plated in four-well chamber slides (Nunc, Naperville, IL) and grown to near confluence. Non-immune mouse immunoglobulin (Ig) G1 antibody in 1:50 dilution was used as a negative control. A counter staining of macrophages was also performed and we did not find any contaminating cells in isolated macrophage (data not shown).

Stroma was collected from the biopsy specimens of the eutopic and ectopic endometrium derived from the women with or without endometriosis. Briefly, specimens obtained at biopsy were placed immediately in a 1:1 mixture of Dulbecco's modified Eagle’s mwedium-F-12 Medium (DMEM-F12; GIBCO BRL, Grand Island, NY) supplemented with $100 \mathrm{U}$ of penicillin per mL (Sigma, 
St. Louis, MO), $100 \mu \mathrm{g}$ of streptomycin per $\mathrm{mL}$ (Sigma), and $2.5 \mu \mathrm{g}$ of amphotericin B per mL (GIBCO BRL). Tissues were then minced into small pieces and incubated for 90 minutes at $37^{\circ} \mathrm{C}$ in a shaking water bath in phosphate-buffered saline (PBS) with 2.5\% collagenase (Wako, Tokyo, Japan), $100 \mathrm{U}$ of penicillin per $\mathrm{mL}, 100 \mu \mathrm{g}$ of streptomycin per $\mathrm{mL}$, and $2.5 \mu \mathrm{g}$ of amphotericin B per $\mathrm{mL}$. The suspension consisted of single stromal cells and fragments of epithelial cells. Cells were pelleted by centrifugation and then were resuspended in fresh medium. After the cell suspension was filtered through a 106- $\mu$ m monofilament nylon mesh, it was washed and resuspended in $10 \mathrm{~mL}$ of prewarmed medium containing $10 \%$ fetal bovine serum.

The cells then were separated from the remaining fragments of epithelial cells by differential sedimentation and adhesion as described previously (24-26). Finally, the cells were resuspended and transferred to the culture dish and then were incubated for 30 minutes at $37^{\circ} \mathrm{C}$ in $95 \%$ air and $5 \% \mathrm{CO}_{2}$. Non-adherent cells were discarded and the attached stromal cells were cultured further with a change of medium every 2-3 days.

The characteristics of the cultured stromal cells were determined by morphological and immunocytochemical studies. The isolated cells were placed in four-chamber slide (Nunc, Naperville, IL). After 24 hours, the slides were washed in PBS, fixed with 4\% paraformaldehyde for 10 minutes, and rinsed with PBS. Slides then were incubated in 0.1\% Triton X-100 for 5 minutes and incubated for 3 hour in $37^{\circ} \mathrm{C}$ as follows: against human cytokeratin monoclonal antibodies (mAb) (epithelial-cell specific) at a dilution of 1:50 (MNF 116;Dako, Denmark), against human vimentin mAb (stromal cell specific) at a dilution of 1:20 (V9; Dako), against human von Willebrand factor mAb (endothelial-cell specific) at a dilution of 1:50 (Dako), and against CD45 mAb (other leukocytes) at a 1:50 (Dako) dilution. 
The specificity of the immunocytochemical staining was confirmed by the deletion of the first antibody. Immunocytochemical staining was performed on at least three different isolated cells with similar results. A counter staining of stroma was also performed to exclude the contamination of epithelial cell or endothelial cell in isolated stromal cell culture (data not shown).

The isolated $\mathrm{M} \phi$ and stroma were serum starved for 24 hour and then were treated with or without lipopolysaccharide (LPS, 5ng/mL) (serotype 0111:B4; Sigma, St. Louis, MO) for an incubation period of another 24 hour. The concentration of HGF in the culture media as conditioned by basal and LPS-stimulated $\mathrm{M} \phi$ and stroma was measured by ELISA.

Statistical analysis. The clinical characteristics of subjects were compared with one-way analysis of variance (ANOVA) and $X^{2}$ test for any difference between two groups. Because concentrations of HGF were not normally distributed, the Mann-Whitney rank sum test or Student's t test was applied for comparison between two groups. For comparisons among three groups, the Kruskal-Wallis test was used to determine the difference among the groups. The results are expressed as either mean \pm standard error of the mean (SEM) or standard deviation (SD). A p $<0.05$ was considered statistically significant.

\section{Results}

Patients' characteristics. The mean ages of control group and women with endometriosis in the PF group were $29.5 \pm 4.7$ years vs. $30.2 \pm 5.6$ years (mean \pm SD), respectively and no differences in age were observed between them. There was also no difference in the mean ages between control women and endometriosis women in the serum group. We found no significant difference in peripheral blood cells, hepatic enzymes and renal function tests between women with or without 
endometriosis in both PF group and serum group (data not shown).

The blood-filled opaque red lesions were classified as polypoid excrescence, ecchymosis, and blood bleb. The nonopaque red lesions were categorized as vesicular bleb and clear papule or clear vesicle. Black lesions comprised of powder burn lesion, blue berry spots or hemosiderin deposits and white lesions included yellowish brown spots or opacifications.

HGF levels in serum and PF depending on staging of endometriosis. We did not find any difference in serum HGF levels between women with endometriosis $(687 \pm 53.3 \mathrm{pg} / \mathrm{mL})$ and control women $(675.1 \pm 40.9 \mathrm{pg} / \mathrm{mL})$. In contrast, PF of women with endometriosis showed a significantly higher concentration of HGF $(1451.7 \pm 90.7 \mathrm{pg} / \mathrm{mL})$ than that of women without endometriosis $(1120.5 \pm 77.3$ $\mathrm{pg} / \mathrm{mL}, \mathrm{p}<0.01$ ) as shown in Figure 1. Kruskal-Wallis test indicated an increased levels of HGF in the secretory phase and menstrual phase in control women and women with endometriosis, respectively ( $<0.05$ for both, PF group).

When we distributed all these women of serum and PF group in different revised-ASRM staging, we didn't find any difference in serum and PF levels of HGF between women with early endometriosis (stage I-II) and advanced endometriosis (stage III-IV) (Figure 2). According to different phases of menstrual cycle, Kruskal Wallis-test again indicated a remarkable increase of HGF in the menstrual phase of women with stage I-II endometriosis, although there was no menstrual phase difference of HGF in women with stage III-IV endometriosis ( $\mathrm{p}<0.05$, Figure 2). This elevated levels of HGF in menstrual phase was only observed for the women in the PF group.

HGF levels in serum and PF depending on morphologic appearance. We divided all samples of endometriosis in serum group and PF group according to the distribution of morphologic 
lesions in pelvic cavity and chocolate cysts. In the serum group, we found a significantly higher concentration of HGF in women containing red lesions $(740 \pm 109.3 \mathrm{pg} / \mathrm{mL})$ than that of either combined black and white lesions $(649 \pm 79.5 \mathrm{pg} / \mathrm{mL}, \mathrm{p}<0.05)$ or chocolate cysts $(485 \pm 43.1 \mathrm{pg} / \mathrm{mL}, \mathrm{p}<0.05)$. After multiple comparisons among these three groups of women distributed by color appearance and chocolate cysts, Kruskal-Wallis test showed that women having opaque or other dominant red lesions released highest levels of HGF in serum ( $<<0.05$, Figure 3$)$.

Again, a marked elevation in the PF concentrations of HGF was observed in women containing red peritoneal lesions $(1685 \pm 183.4 \mathrm{pg} / \mathrm{mL})$ comparing to women having combined black and white lesions $(1224 \pm 67.8 \mathrm{pg} / \mathrm{mL}, \mathrm{p}<0.05)$ and also to women containing chocolate cysts $(1118 \pm 83.1$ $\mathrm{pg} / \mathrm{mL}, \mathrm{p}<0.01$, Figure 3). Multiple comparisons among these three groups of women by using Kruskal-Wallis test demonstrated the highest levels of HGF in PF of women who harbored red lesions in pelvic cavity ( $<<0.05)$. These increased levels of HGF in women containing red lesions (both serum and PF group) were also significantly higher than that of women without endometriosis (data not shown).

There was no woman in the menstrual phase in serum group. We excluded eight cases of menstrual phase in PF group of endometriosis due to their inhomogenous distribution according to color appearances and chocolate cysts. No apparent differences in serum HGF levels were noticed between proliferative phase and and secretory phase among these three groups of women (Figure 3). However, a significant elevation of HGF in PF was observed in the secretory phase of women containing red lesions $(p<0.05)$ and in the proliferative phase of women having chocolate cysts $(p<0.05$ Figure 3$)$.

Production of HGF by basal and LPS-stimulated PF macrophages, endometrial and endometriotic stroma. In order to verify our elevated results of HGF in PF, we examined the production 
of HGF by the isolated $\mathrm{M} \phi$ derived from the PF and endometrial and endometriotic stroma derived from women with or without endometriosis. We found that both basal and LPS-treated stroma were the main source of HGF production than that of $\mathrm{M} \phi$. We determined the dose of LPS $(5 \mathrm{ng} / \mathrm{mL})$ from a previously described dose-dependent study of LPS (25). In fact, both basal and stimulated-stroma from the eutopic endometrium produced a 3-4 times higher concentration of HGF than that of M $\phi$ (Figure 4). This production of HGF by stroma was more remarkable in women with endometriosis than that of non-endometriosis $(\mathrm{p}<0.01)$. In contrast, a small but a significant amount of HGF was also produced by the peritoneal $\mathrm{M} \phi$ of women with endometriosis than that of non-endometriosis $(\mathrm{p}<0.05)$ (Figure 4). Although there was no variation in the production of HGF by the basal M $\phi$ between women with or without endometriosis, a significant increase in the secretion of HGF by the basal stroma was observed in women with endometriosis than that in non-endometriosis $(\mathrm{p}<0.01)$.

When we examined HGF production by the stroma of pelvic endometriosis and stroma of chocolate cysts derived from a fraction of these women, we found that stroma derived from chocolate cysts produced a significantly less concentrations of HGF than that derived from blood-filled opaque lesions of pelvic endometriosis. However, there was no difference in HGF production between stroma derived from ectopic endometrium and eutopic endometrium of women with pelvic endometriosis (data not shown).

\section{Discussion}

The revised-ASRM classification of endometriosis still retains some controversy regarding score-based staging of endometriosis and activity of disease. The advanced staging of revised-ASRM classification included the presence of variable sizes of ovarian endometrioma and coexistent extension 
of fibrosis in pelvic cavity. However, there is no information in this revised-ASRM classification about the lesion activity of endometriosis that may induce an inflammatory response in pelvic cavity and may be associated with infertility or pelvic pain of women containing active lesions of endometriosis. Our current study described an example of this important issue. We demonstrated parallel measurement of HGF in serum and PF of women with or without endometriosis and found that elevation in levels of HGF in both serum and PF were not associated with either early or advanced staging of endometriosis. Instead, PF levels of HGF can differentiate women with endometriosis from non-endometriosis. In addition, we found that women containing blood-filled red morphologic lesions of endometriosis produced a significantly higher concentration of HGF in both serum and PF comparing to that of women containing other peritoneal lesions or chocolate cysts. The possible source of this elevated HGF in PF was demonstrated by an in vitro experimental study showing that stromal cells derived from eutopic or ectopic endometrium or the infiltrated $\mathrm{M} \phi$ in PF may be the potential source of HGF in pelvic microenvironment.

Our results further demonstrated that red color appearance of endometriosis especially the blood-filled opaque red lesions represent the most active stage of the disease that may incite a stronger pelvic inflammatory response and may result in consequent accumulation of HGF in serum and peritoneal fluid. We previously reported with a small number of samples that early staging and women containing dominant red peritoneal lesions were associated with increased concentrations of HGF in PF (18). Although we did not find any difference of HGF levels between early and advanced staging of endometriosis in our current study, women in the menstrual phase of early endometriosis and highly active red morphologic lesions of endometriosis manifested the similar results of HGF in PF as we 
described previously (18). After adjusting different variables in women with endometriosis, we found that active morphologic lesions rather than staging of endometriosis were independently associated with the increased concentrations of HGF in the PF.

Our findings of increased HGF concentrations in PF are in agreement with those of other published reports $(27,28)$ who demonstrated a similar elevation of other cytokines in women with endometriosis. In some recent studies (29-31), higher IL-6, IL-8 and TNF-alpha concentration in PF were found to be associated with active lesions of endometriosis. Since, HGF promoter retains the response elements for all these cytokines including estradiol (32, 33), we speculate that a synergistic action between cytokines and steroid hormone in pelvic microenvironment may stimulate the endometrial cells in eutopic or ectopic endometrium to produce and release HGF in PF. An interaction between innate immune system and ovarian steroid hormones with the consequent production of different cytokines including HGF has been recently described (34). In fact, we already reported a parallel increase of HGF and estradiol in the PF of women with early endometriosis and in women having dominant distribution of red lesions in pelvic cavity (18). In addition, active red lesions have been recognized as peritoneal lesions with higher mitogenic and angiogenic activity $(14,28)$.

In our recent study by Khan et al. (35), we found a parallel increase in the immunoexpressions of HGF and c-Met in the eutopic endometrium of women who also harbored active red lesions. These results indicated that eutopic and ectopic endometrium are mutually responsible for the increased production of HGF and their increased accumulation in PF as shown in our current study. Our results of HGF content in PF do not agree with that of Osuga et al. (15) who found that HGF concentration was higher in stage III-IV endometriosis, intermediate in stage I-II endometriosis, and least in control women. 
Again, Zong et al. (17) demonstrated that degree of endometriosis was positively correlated with HGF concentration in PF and serum with a linear correlation between PF and serum levels of HGF. There is no doubt that women with endometriosis produce an increase amount of HGF in PF than that of control women, however, both of these studies did not clarify the coexistence of red pigments or chocolate cysts in their grouping of patients that could overestimate their results of HGF in advanced endometriosis and less increase in early endometriosis. We did not find any positive correlation between PF and serum levels of HGF. This could be due to small number of cases in our serum group. Instead, we found a significantly higher concentration of HGF in both PF and serum of women containing blood-filled opaque red lesions in pelvic cavity.

The increased generation and extension of blood vessels in active red lesions may result in the possible entry of HGF into the systemic circulation from eutopic or ectopic endometrium and their consequent elevation in serum. Since the concentration of HGF in PF was double than that of serum, we speculate that elevated serum concentration of HGF in women harboring red lesions was secondary to the local production of HGF by active pelvic endometriotic lesions and their consequent release into the circulation.

Our results of HGF in serum and PF correspond to the results of isolated $\mathrm{M} \phi$ and stroma in primary culture. Since endometriosis induces an inflammatory response in pelvic microenvironment (25, 36), we observed that the peritoneal $\mathrm{M} \phi$ in response to an inflammatory mediator (LPS) also produce a small amount of HGF in pelvic environment. This secretion of HGF by $\mathrm{M} \phi$ coupled with its increased production by stroma of endometriosis could elevate the concentrations of HGF in the PF and their consequent entry into circulation. The increased production of HGF by the cells of mesenchymal origin is 
well described. We also found that stromal cells of both eutopic and ectopic endometrium of women with endometriosis are the main source of HGF. The production of HGF by basal stroma was further elevated in response to stimulation with LPS.

Although data not shown, it was interesting to observe that stromal cells derived from peritoneal lesions and their corresponding eutopic endometrium equally produce increased concentration of HGF in the culture media, however, stroma derived from chocolate cyst generate less amount of HGF. Further studies are required to confirm our current findings. We assume that HGF in PF derives largely from eutopic and ectopic endometrial tissues and is involved in the growth and progression of freshly implanted peritoneal lesions. This could constitute a substantial percentage of HGF in serum during menstrual phase as reported by Negami et al. (37).

In conclusion, our results suggest that increased HGF levels in either serum or PF failed to differentiate women between early and advanced endometriosis as recommended by revised-ASRM classification. Instead, it is the red color appearance of endometriosis in pelvic cavity and the local production of HGF that were associated with elevated concentrations of HGF in both serum and PF. These results correspond to the activity of endometriosis rather than extension of disease as described by r-ASRM staging. Although it is premature to use HGF as a non-surgical tool to predict the activity of endometriosis, a multi-center study collecting large samples are needed to establish the clinical application of our current result. Again, our results further emphasize the importance in finding necessary parameters to elucidate the activity of endometriosis and their possible involvement in infertility. 


\section{Acknowledgement}

We thank Miss. Kazumi Hayashida and Miss. Kyoko Ishida, Department of Obstetrics and Gynecology, for their excellent technical assistance in sampling preservation. We are also grateful to Dr. Kondo Hisayoshi, Department of Bio-Statistics, and Dr. Tetsushi Hamasaki, Department of Obstetrics and Gynecology, Nagasaki University School of Medicine, Nagasaki, Japan for their technical assistance in statistical analysis. 


\section{References}

1) Miyazawa K, Tsubouchi H, Naka D, Takahashi K, Okigaki M, Arakaki N, et al. Molecular cloning and sequence analysis of cDNA for human hepatocyte growth factor. Biochem. Biophys. Res. Commun. 1989;163: 967-73.

2) Nakamura T, Nishizawa T, Hagiya M, Seki T, Shimonishi,H, Sugimura A, et al. Molecular cloning and expression of human hepatocyte growth factor. Nature 1989;342:440-443.

3) Wiedner KM, Arakaki N, Vandekereckhove J, et al. Evidence for the identity of human scatter factor and human hepatocyte growth factor. Proc Natl Acad Sci USA 1991;88:7001-05.

4) Nakamura T, Teramoto H, Ichihara A. Purification and characterization of a growth factor from rat platelets from mature parenchymal hepatocytes in primary cultures. Proc. Natl. Acad. Sci. USA 1986;83:6489-93.

5) Tajima H, Matsumoto K, Nakamura T. Regulation of cell growth and motility by hepatocyte growth factor and receptor expression in various cell species. Exp Cell Res 1992;20:423--431.

6) Wagatsuma S, Konno R, Sato S, Yajima A. Tumor angiogenesis, hepatocyte growth factor, and c-Met expression in endometrial carcinoma. Cancer 1998;82:520-530.

7) Sonnenberg E, Meyer D, Weidner KM, Birchmeier C. Scatter factor/hepatocyte growth factor and its receptor, c-met tyrosine kinase, can mediate a signal exchange between mesenchyme and epithelia during mouse development. J Cell Biol 1993;123:223-235.

8) Sugawara J, Fukaya T, Murakami T, Yoshida H, Yajima A. Hepatocyte growth factor stimulates proliferation, migration, and lumen formation of human endometrial epithelial cells in vitro. Biol Reprod 
1997;57:936-942.

9) Fujishita A, Nakane PK, Koji T, Masuzaki H. Chavez RO, Yamabe T, Ishimaru T. Expression of estrogen and progesterone in endometrium and peritoneal endometriosis: an immunohistochemical and in situ hybridization study. 1997;67:856-864.

10) Nisolle M, Casanas-Rouz F, Donnez J. Immunohistochemical analysis of proliferative activity and steroid receptor expression in peritoneal and ovarian endometriosis. 1997;68:912-919

11) Tsudo T, Harada T, Iwabe T, Tanikawa M, Nagano $Y$, Ito M, et al. Altered gene expression and secretion of interleukin-6 in stromal cells derived from endometriotic tissues. Fertil. Steril. 2002;73:205-211.

12) Tseng JF, Ryan IP, Milam TD, Murai JT, Schriock ED, Landers DV, et al. Interleukin-6 secretion in vitro is upregulated in ectopic and eutopic endometrial stromal cells from women with endometriosis. J Clin Endocrinol Metab., 1996;81:1118-22.

13) McLaren J, Prentice A, Charnock-Jones DS Smith SK. Vascular endothelial growth factor (VEGF) concentrations are elevated in peritoneal fluid of women with endometriosis. Hum Reprod 1996;11:220-223.

14) Donnez J, Smoes P, Gillerot S, Casanas-Roux F, Nissole M. Vascular endothelial growth factor in endometriosis. Hum. Reprod. 1998;3:1686-690.

15) Osuga Y, Tsutsumi O, Okagaki R, Takai Y, Fujimoto A, Suenaga A, et al. Hepatocyte growth factor concentrations are elevated in peritoneal fluid of women with endometriosis. Hum. Reprod. 1999;14:1611-613. 
16) Mahnke JL, Dawood Y, and Huang JH. Vascular endothelial growth factor and interleukin-6 in peritoneal fluid of women with endometriosis. Fertil Steril., 2000;73:166-170.

17) Zong LL. Li YL, Ha XQ. Determination of HGF concentration in serum and peritoneal fluid in women with endometriosis. Di Yi Jun Da Xue Xue Bao. 2003;23(8):757-760 (Chinese).

18) Khan KN, Masuzaki H, Fujishita A, Hamasaki T, Kitajima M, Hasuo A, Miyamura Y, Ishimaru T. Association of interleukin-6 and estradiol with hepatocyte growth factor in peritoneal fluid of women with endometriosis. Acta Obstet Gynecol Scand., 2002;81(8):764-771.

19) Kitajima M, Khan KN, Fujishita A, Masuzaki H, Ishimaru T. Association between Chlamydia trachomatis infection and occurrence of endometriosis. Obstetrics and Gynecological Practice 2003;52(2):245-250 (Japanese).

20) Miura S, Khan KN, Fujishita A, Kitajima M, Samejima T, Masuzaki H, Ishimaru T. Macrophage infiltration and prostaglandin production by different uterine leiomyomas. The $57^{\text {th }}$ Annual Congress of the Japan Society of Obstetrics and Gynecology P2-150 (abstract).

21) The American Society for Reproductive Medicine. Revised American Society for Reproductive Medicine classification of endometriosis: 1996. Fertil. Steril. 1997;67:817-821.

22) Jansen, RPS and Russel P. Non-pigmented endometriosis: clinical, laparoscopic and pathologic definition. Am J. Obstet Gynecol., 1996;155:1154-1159.

23) Khan KN, Masuzaki H, Fujishita A, Kitajima M, Sekine I, Ishimaru T. Higher activity by opaque endometriotic lesions than non-opaque lesions. Acta Obstet Gynecol Scand, 2004;83:375-382.

24) Osteen KG, Hill GA, Hargrove JT, Gorstein F. Development of a method to isolate and culture highly purified populations of stromal and epithelial cells from human endometrial biopsy specimens. 
Fertil Steril 1989;52:965-972.

25) Khan KN, Masuzaki H, Fujishita A, Kitajima M, Kohno T, Sekine I, Matsuyama T, Ishimaru T. Regulation of hepatocyte growth factor by basal and stimulated macrophages in women with endometriosis. Hum Reprod, 2005;20:49-60.

26) Rana N, Braun DP, House R. Basal and stimulated secretion of cytokines by peritoneal macrophages in women with endometriosis. Fertil Steril 1996;65:925-930.

27) Punnonen J, Teisala K, Ranta H. Increased levels of interleukin-6 and interleukin-10 in the peritoneal fluid of patients with endometriosis. Am J Obstet Gynecol., 1996;174:1522-1526.

28) Fujishita A, Hasuo A, Khan KN, Masuzaki H, Nakashima H, Ishimaru T. Immunohistochemical study of angiogenic factors in endometrium and endometriosis. Gynecol. Obstet. Invest. 1999;48(suppl 1):36-44.

29) Harada T, Yoshioka H, Yoshida S, Iwabe T, Oonohara Y, Tanikawa M, et al. Increased interleukin-6 levels in peritoneal fluid of infertile patients with active endometriosis. Am J Obstet Gynecol., 1997;176:593-597.

30) Arici A, Tazuke SI, Attar E, Kliman HJ, Olive DL. Interleukin-8 concentration in peritoneal fluid of patients with endometriosis and modulation of interleukin-8 expression in human mesothelial cells. Mol Hum Reprod, 1996; 2:40-45.

31) Taketani Y, Kuo TM, Mizuno M. Comparison of cytokine levels and embryo toxicity in peritoneal fluid in infertile women with untreated or treated endometriosis. Am J Obstet Gynecol $1992 ; 167: 265-270$. 
32) Zarnegar R. Regulation of HGF and HGFR gene expression. Epithelial-mesenchymal interactions in cancer 1995;74: 33-49.

33) Liu Y, Michalopaulos GK, and Zarnegar R. Structural and functional characterization of the mouse hepatocyte growth factor gene promoter. J Biol Chem. 1994;269:4152-4160.

34) Khan KN, Masuzaki H, Fujishita A, Kitajima M, Sekine I, Matsuyama T, Ishimaru T. Estrogen and progesterone receptor expression in macrophages and regulation of hepatocyte growth factor by ovarian steroids in women with endometriosis. Hum Reprod 2005 (in press).

35) Khan KN, Masuzaki H, Fujishita A, Kitajima M, Sekine I, Ishimaru T. Immunoexpression of hepatocyte growth factor and c-Met receptor in eutopic endometrium predicts the activity of ectopic endometrium. Fertil Steril, 2003;79:173-181.

36) Khan KN, Masuzaki H, Fujishita A, Kitajima M, Sekine I, Ishimaru T. Differential macrophage infiltration in early and advanced endometriosis and adjacent peritoneum. Fertil Steril, 2004;81:652-661. 37) Negami AI, Sasaki H, Kawakami Y, Kamitani N, Kotsuji F, Tominaga T, et al. Serum human hepatocyte growth factor in human menstrual cycle and pregnancy: a novel serum marker of regeneration and reconstruction of human endometrium. Horm. Res. 1995;44(suppl 2):42-46. 


\section{Figure Legends}

Figure 1. Shows the serum and peritoneal fluid (PF) concentration of hepatocyte growth factor (HGF) in women with endometriosis and control women and according to different phases of menstrual cycle. A significantly higher concentration of HGF in PF was found in women with endometriosis than that of control women ( ${ }^{* *} \mathrm{p}<0.01$ vs. control, total cases). ${ }^{*} \mathrm{p}<0.05$ vs. proliferative phase in the control group; ${ }^{*} \mathrm{p}<0.05$ vs. either proliferative phase or secretory phase in endometriosis group (multiple comparison by Kruskal-Wallis test).

Figure 2. Shows the distribution of hepatocyte growth factor (HGF) levels in serum and peritoneal fluid (PF) of women with endometriosis according to the revised-ASRM classification of endometriosis and different phases of menstrual cycle. No difference in serum and PF levels of HGF was observed between women with early endometriosis (stage I-II) and advanced endometriosis (stage III-IV). ${ }^{*} \mathrm{p}<0.05$ vs. either proliferative phase or secretory phase (multiple comparison by Kruskal-Wallis test).

Figure 3. Shows the serum and peritoneal fluid (PF) levels of hepatocyte growth factor (HGF) in women with endometriosis and according to the distribution of red lesions, combined black and white lesions and chocolate cysts. Women containing red lesions in both serum and PF had significantly higher concentrations of HGF than those containing combined black and white lesion or women containing chocolate cysts ( ${ }^{*} \mathrm{p}<0.05$ for both serum and PF group, multiple comparison by Kruskal-Wallis test). ${ }^{* *} \mathrm{p}<0.05$ vs. proliferative phase in women containing red lesions; ${ }^{\mathrm{p}} \mathrm{p}<0.05$ vs. secretory phase in women containing chocolate cysts. 
Figure 4. Shows the production of HGF in the culture media as conditioned by the basal and lipopolysaccharide (LPS, 5ng/mL)-stimulated macrophages ( $\mathrm{M} \phi$ ) derived from the peritoneal fluid (A) and stroma derived from the eutopic endometrium (B) of women with or without endometriosis. A. ${ }^{*} \mathrm{p}<0.05$ vs. non-endometriosis. B. ${ }^{* *} \mathrm{p}<0.01$ vs. non-endometriosis (non-treated $\mathrm{M} \phi$ ); $\quad{ }^{*} \mathrm{p}<0.01$ vs. non-endometriosis (LPS-treated M $\phi$ ). The results are expressed as mean \pm SEM of three different experiments derived from three separate women with or without endometriosis. 
Figure 1

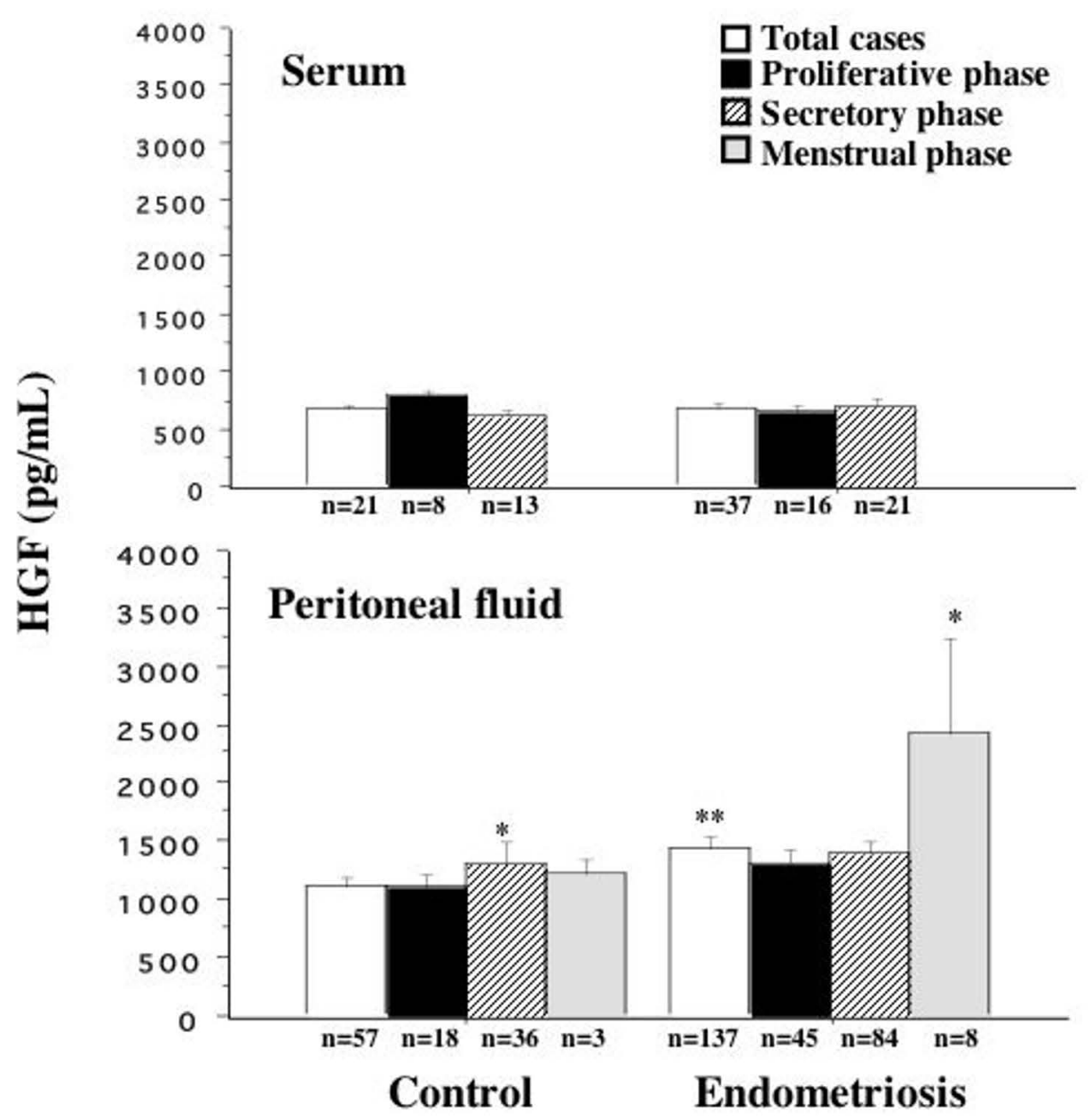


Figure 2

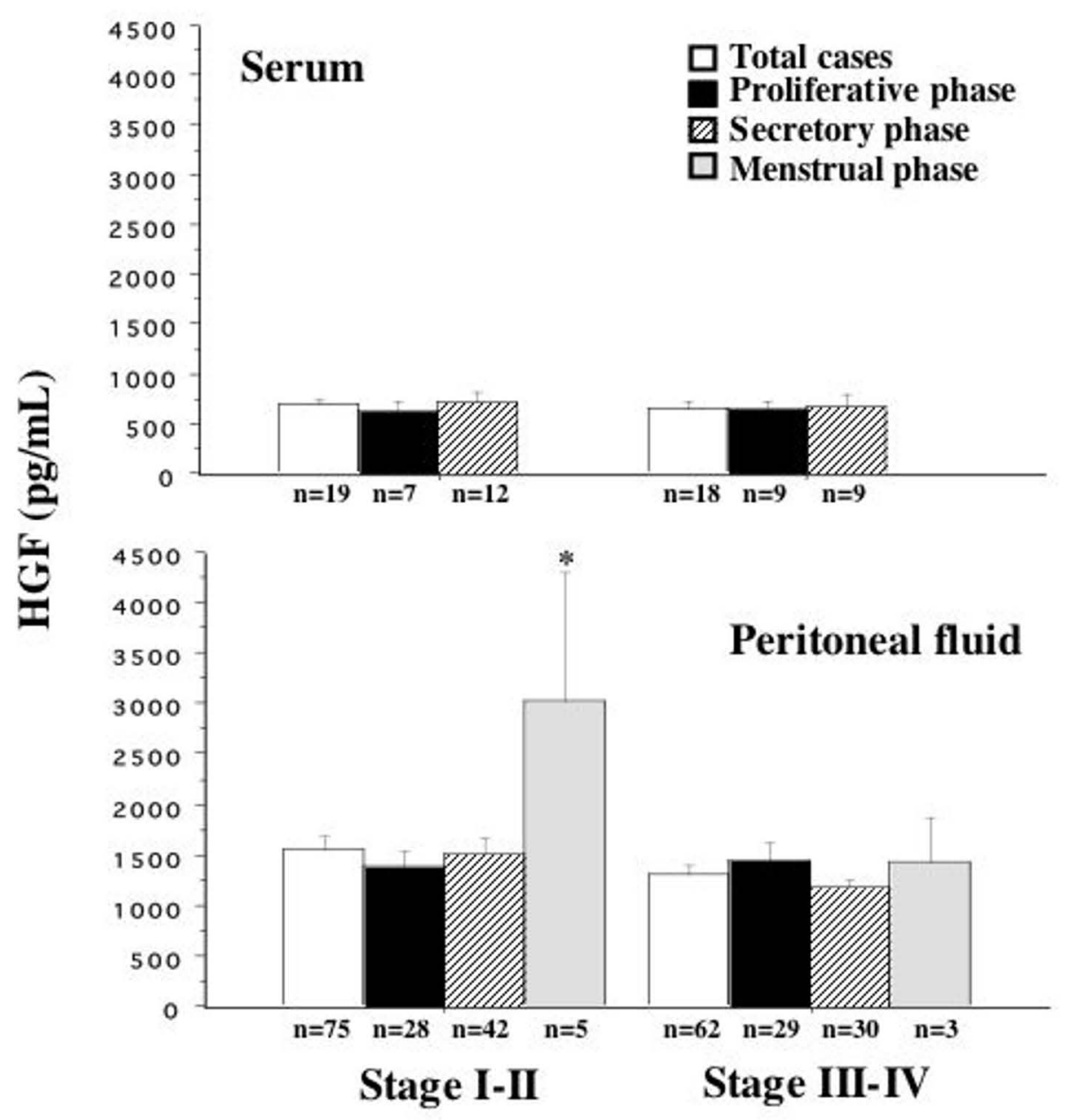


Figure 3

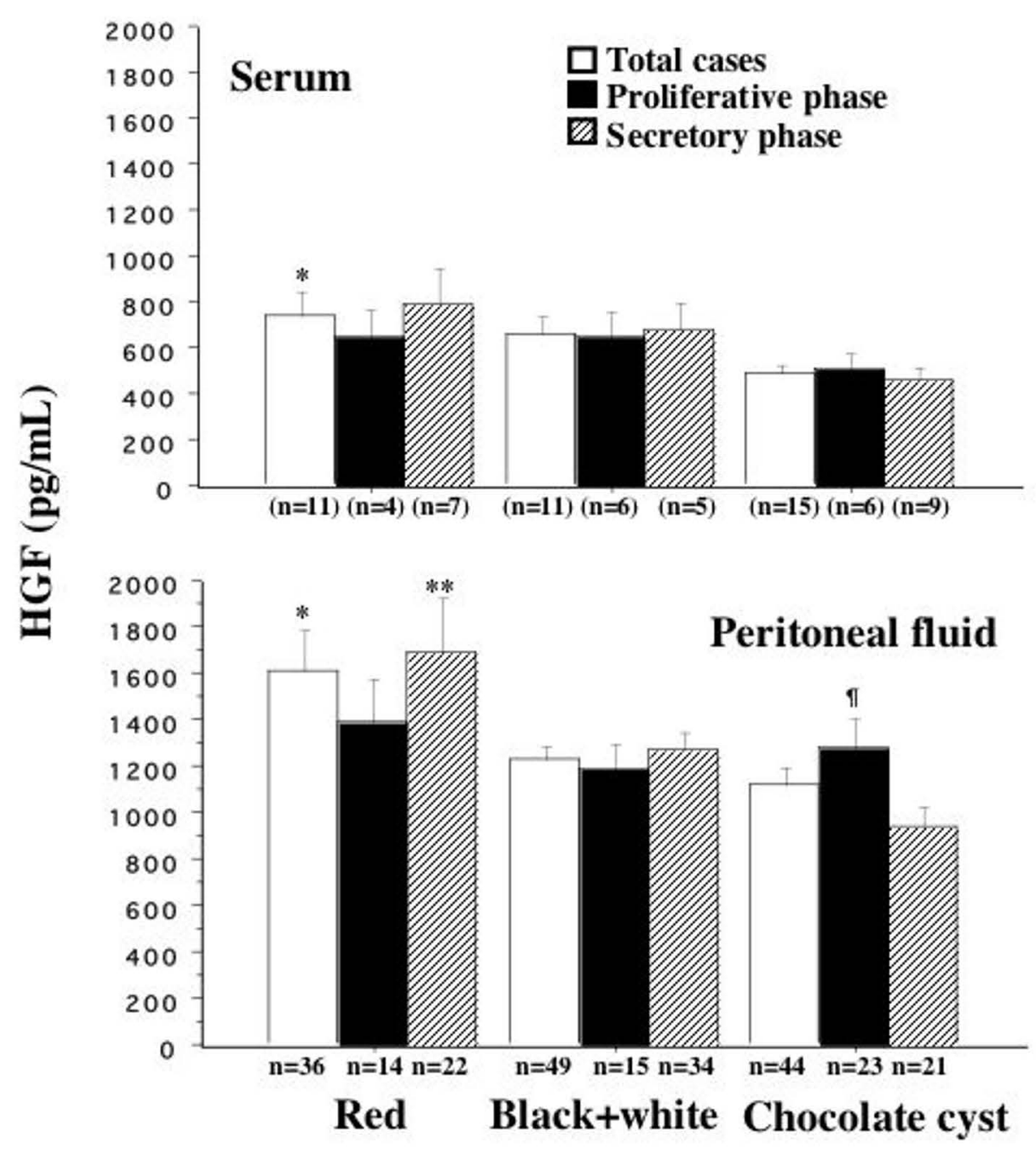


Figure 4

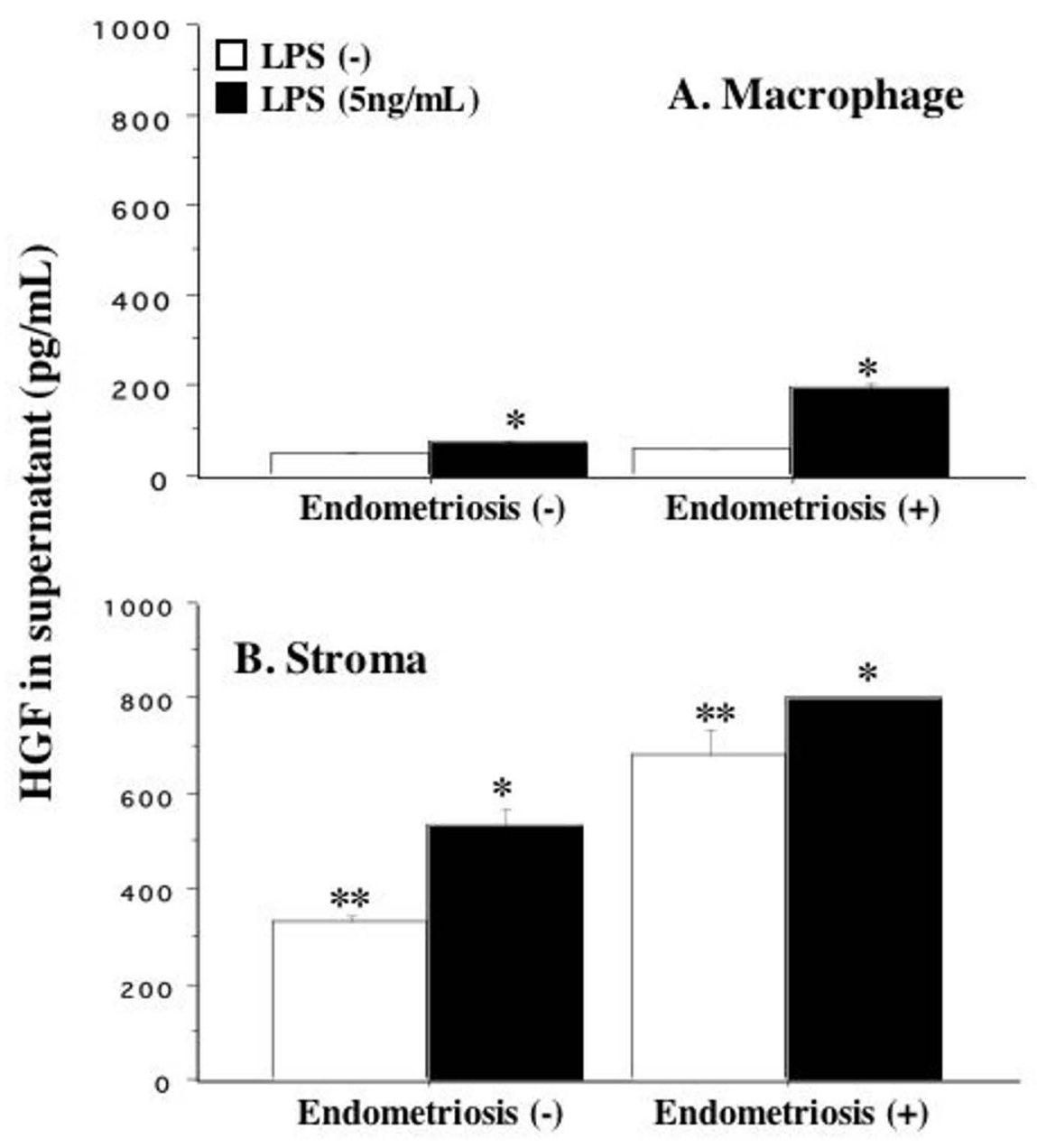

